\title{
Intraoperative neurophysiological monitoring during scoliosis surgery in patients with Duchenne muscular dystrophy
}

\author{
S. E. Dulfer ${ }^{1}$ F. Lange ${ }^{2}$ - F. H. Wapstra ${ }^{3}$ - A. R. E. Potgieser ${ }^{1}$ - J. P. Valk ${ }^{4} \cdot$ A. R. Absalom ${ }^{4} \cdot$ R. J. M. Groen ${ }^{1} \cdot$ C. Faber ${ }^{3}$. \\ G. Drost ${ }^{1,2}$
}

Received: 26 August 2019 / Accepted: 10 May 2020 / Published online: 21 May 2020

(c) The Author(s) 2020

\begin{abstract}
Purpose Little is known about the reliability and value of intraoperative neurophysiological monitoring (IONM) in patients with Duchenne muscular dystrophy (DMD) undergoing scoliosis correction surgery. The aim of this study was to investigate the feasibility of IONM and the cortical excitability in these patients.

Methods Fifteen patients with DMD and scoliosis and 15 patients with adolescent idiopathic scoliosis (AIS) underwent scoliosis correction surgery with the use of IONM. IONM consisted of transcranial electrical stimulation motor evoked potential (Tc-MEP) and somatosensory evoked potential (SSEP) monitoring. The highest Tc-MEP amplitudes were collected to test the feasibility. Preoperative compound muscle action potentials (CMAPs) and transcranial magnetic stimulation (TMS)-MEPs were recorded to test the cortical excitability. SSEPs were scored as elicitable or not elicitable.

Results Tc-MEP amplitudes were significantly lower in the DMD group for both the gastrocnemius and tibialis anterior muscles. However, the abductor hallucis muscle had similar amplitudes in both the DMD as the AIS group. TMS/CMAP and Tc-MEP/CMAP ratios were similar in the DMD and AIS group ( $P=0.126$ and $P=0.792$ respectively).

Conclusions Tc-MEP and SSEP monitoring is feasible, particularly when Tc-MEPs are recorded from the abductor hallucis muscle in patients with DMD. Similar TMS/CMAP and Tc-MEP/CMAP ratios show that there were no differences observed in cortical excitability between the groups. IONM seems a feasible and valuable neurophysiological tool to signal possible surgically induced damage to the spinal cord during scoliosis correction surgery in patients with DMD.
\end{abstract}

Keywords Duchenne muscular dystrophy - Intraoperative neurophysiological monitoring · Transcranial electrical stimulation motor evoked potentials $\cdot$ Scoliosis correction surgery $\cdot$ Cortical excitability

\section{Introduction}

Duchenne muscular dystrophy (DMD), a recessive X-linked muscle disease, is the most common of the muscular dystrophies. It is caused by mutations in the dystrophin gene which encodes dystrophin, an important structural component of muscle tissue. Loss of dystrophin causes progressive muscle weakness, with the proximal muscles more affected than the distal muscles [1]. During puberty, patients are at risk of developing scoliosis due to weakness of the spinal muscles in combination with rapid growth of the spinal column. Lebel et al. followed up patients with DMD for 15 years and found that $20 \%$ develop scoliosis and need spinal deformity surgery [2]. The goal of scoliosis correction surgery is to improve pulmonary function, decrease pain and improve wheelchair and seat positioning [3, 4]. The risks of surgery for these patients are, however, significant, and the decision 
whether or not to operate is often quite difficult to make, especially as there is no convincing evidence that these patients benefit from the procedure [5-7]. However, despite the lack of randomized controlled trials, scoliosis surgery is still recommended since it improves sitting balance and tolerance, pain and quality of life [3, 8].

Intraoperative neurophysiological monitoring (IONM) has proven its value in predicting and preventing iatrogenic neurological deficits in spine surgery $[9,10]$. However, monitoring patients with DMD provides multiple challenges, and moreover, they have an increased risk of spinal cord injury during scoliosis surgery $[11,12]$. Therefore, reliable monitoring of spinal cord function during scoliosis correction surgery is important in this category of patients.

One challenge with monitoring patients with DMD is that to measure transcranial electrical stimulation motor evoked potentials (Tc-MEPs), muscle excitation is necessary. Because of muscle degeneration, it can be challenging to obtain reliable Tc-MEP measurements.

A second factor that may disturb IONM in patients with DMD is that the cortical excitability might be different from that found in other patients, due to reduced dystrophin levels in the central nervous system (CNS) [13]. This CNS involvement of dystrophin suggests that IONM measurements might be interpreted differently as compared to patients without reduced dystrophin in the CNS.

Little is known about scoliosis correction surgery with the use of SSEPs and Tc-MEPs in patients with DMD since there are only few cases described in the literature [14-18]. Therefore, we investigated (1) the feasibility of IONM and (2) the cortical excitability in patients with DMD and scoliosis.

The goal of the study was to investigate and compare cortical excitability and feasibility of IONM in patients with DMD-associated scoliosis and a cohort of patients with adolescent idiopathic scoliosis (AIS).

\section{Materials and methods}

\section{Subjects}

This retrospective study from 2003 to 2017 included data from 15 patients with DMD and scoliosis and 15 patients with AIS. Patient characteristics are listed in Table 1. Patients with DMD had become wheelchair dependent at a mean age of 10.5 years $(S D \pm 2.2)$ and before scoliosis surgery was performed. DMD was diagnosed by genetic testing or muscle biopsy.

\section{Surgical technique}

All surgical procedures were performed via a posterior approach with the patient in prone position. Pedicle screws were inserted with the use of computer-assisted navigation. Scoliosis was corrected by conventional operating techniques at the discretion of the operating surgeon. For definitive fixation, dual posterior rods were mounted on pedicle screws. In order to ensure longevity of the fixation, extensive posterior bone grafting was performed.

\section{Anesthetics}

All patients were screened by an anesthesiologist before surgery. A central venous line was inserted in all DMD patients. All patients received total intravenous anesthesia with propofol, and remifentanil or sufentanil. Muscle relaxants were only given prior to intubation to avoid the negative effect of muscle relaxation on the muscle responses. Esketamine was sometimes used perioperatively as a bolus or by infusion as an analgesic adjuvant. To help manage the intraoperative blood loss, the use of a cell-saver system and tranexamic acid was considered.

\begin{tabular}{lllc}
\hline & $\begin{array}{l}\text { Duchenne muscular } \\
\text { dystrophy }(N=15)\end{array}$ & $\begin{array}{l}\text { Adolescent idiopathic } \\
\text { scoliosis }(N=15)\end{array}$ & $P$-value \\
\hline Age at surgery (Mean \pm SD years) & $15.4 \pm 1.6$ & $15.7 \pm 2.4$ & 0.567 \\
Male (\%) & 100.0 & 20.0 & $<0.001^{*}$ \\
Surgery time (minutes) & $497.20 \pm 86.68$ & $344.40 \pm 79.38$ & $<0.001^{*}$ \\
Duration of hospitalization (days) & $14.20 \pm 10.05$ & $8.27 \pm 2.46$ & $<0.01^{*}$ \\
Main cobb-angle preoperative (Mean $\pm \mathrm{SD})$ & $50.1^{\circ} \pm 16.8^{\circ}$ & $63.9^{\circ} \pm 11.2^{\circ}$ & $<0.05^{*}$ \\
Main cobb-angle postoperative $(M e a n \pm S D)$ & $36.2^{\circ} \pm 18.8^{\circ}$ & $30.7^{\circ} \pm 8.9^{\circ}$ & 0.539 \\
\hline
\end{tabular}

*Significant $p<0.05$

$S D$ standard deviation; $N$ number 


\section{Intraoperative neurophysiological evaluation}

\section{Transcranial electrical stimulation motor evoked potential}

Intraoperative Tc-MEPs were performed according to a standard procedure using a custom-made constant voltage stimulator (before 8-2015, NeuroGuard@), JS Center, Bedum, The Netherlands; after 8-2015, NIM-Eclipse E4 IONM system, Medtronic BV, The Netherlands). Supramaximal stimulation was performed in which the voltage was optimized per patient. Voltages varied between patients from 110 to $400 \mathrm{~V}$ in the DMD group and ranged from 80 to $300 \mathrm{~V}$ in the AIS group. These large differences in voltage are due to the different pulse durations that were used. A higher voltage (250-400 V) corresponded with a pulse duration of $75 \mu \mathrm{s}$, and the lower voltages (80-130 V) corresponded with a pulse duration of $300 \mu$ s. The number of pulses were optimized per patient and varied from 4 to 7 pulses. Interstimulus intervals, ranging from 0.2 to $1 \mathrm{~ms}$, were optimized per patient as well.

Tc-MEPs were recorded using surface electrodes $(3 \mathrm{M} \circledast$ ECG) at the left and right tibialis anterior (TA), the left and right gastrocnemius (GAS), the left and right abductor hallucis (AH) and the right or left hand. From October 2013, Tc-MEP recordings in the patients with DMD were also performed in non-limb muscles consisting of the sphincter muscles, using needle electrodes, and the left and right rectus abdominis.

A decrease of $80 \%$ of MEP amplitude was considered as warning criteria of potential neurological damage, but only if anesthetic, physiological or technical reasons for the decline were ruled out.

\section{Somatosensory evoked potentials}

SSEPs were recorded after stimulation of the posterior tibial nerves. A distinction was made between the number of SSEPs for the left and right leg. The left and/or right ulnar or median nerve was also stimulated for SSEPs. Stimulation was performed with an intensity up to $60 \mathrm{~mA}$, a duration of $0.2 \mathrm{~ms}$ and a $4.3 \mathrm{~Hz}$ repetition rate. The recordings were performed on the scalp at $\mathrm{Cz}-\mathrm{Fz}$.

\section{Preoperative neurophysiological evaluation}

\section{Compound muscle action potentials}

Compound muscle action potential amplitudes (CMAPs) were recorded from the TA muscle after stimulating the fibular nerve (Nicolet Synergy EP/EMG system, FMH Medical BV, The Netherlands). CMAPs were recorded using 1-cm-diameter surface electrodes for both left and right TA muscles. Stimulation was performed with a current up to $100 \mathrm{~mA}$ and a pulse duration of $1 \mathrm{~ms}$.

\section{Transcranial magnetic stimulation}

Transcranial magnetic stimulation (TMS) was performed with a magnetic stimulator connected to a round coil at anatomical location Fz-Cz (Magstim $200 \mathrm{HP}$, the Magstim Comp, Dyfed, UK). Single pulses were applied at $100 \%$ stimulation.

\section{Statistical analysis}

CMAP, TMS, Tc-MEP and SSEP data were compared between the patients with DMD and patients with AIS. TMS/CMAP and Tc-MEP/CMAP ratios were calculated. The patients characteristics were also compared between these groups. Due to our small sample sizes, the Shapiro-Wilk test was used, to test whether the results were normally distributed. Normally distributed data were analyzed with the independent T-test. Not normally distributed data were analyzed with the Mann-Whitney U test.

\section{Results}

\section{Intraoperative Tc-MEPs and SSEPs}

Fifteen patients with DMD for whom IONM data were available were included. In all 15 patients with DMD (100\%), Tc-MEPs were elicitable during surgery. There were no decreases in Tc-MEP amplitude of more than $80 \%$. No DMD patients deteriorated neurologically directly postoperatively. Tc-MEPs were elicitable in all patients with AIS (100\%).

The number of muscles that were suitable for Tc-MEP monitoring in patients with DMD was limited. At the start of surgery, in all 15 DMD patients, Tc-MEPs were elicitable from at least one leg muscle, and in 13 DMD patients $(86.7 \%)$ responses were elicitable from five or more muscles. In all 15 DMD patients, Tc-MEPs were elicitable in at least two muscles at the end of surgery, and in 14 DMD patients (93.3\%) Tc-MEPs were elicitable in five or more muscles at the end of surgery. No patients had fewer muscles that were elicitable with Tc-MEP at the end of surgery than at the start of surgery. The Tc-MEPs of the patients with AIS were always elicitable in all six muscles.

The highest Tc-MEP amplitude per muscle was scored. The median and interquartile range of the amplitudes were calculated and are shown in $\mathrm{mV}$ per muscle for both patients with DMD and AIS in Fig. 1 and Table 2. The TA left and TA right, and the GAS left and GAS right were significantly different $(p<0.003)$ as evidenced by the Mann-Whitney U 


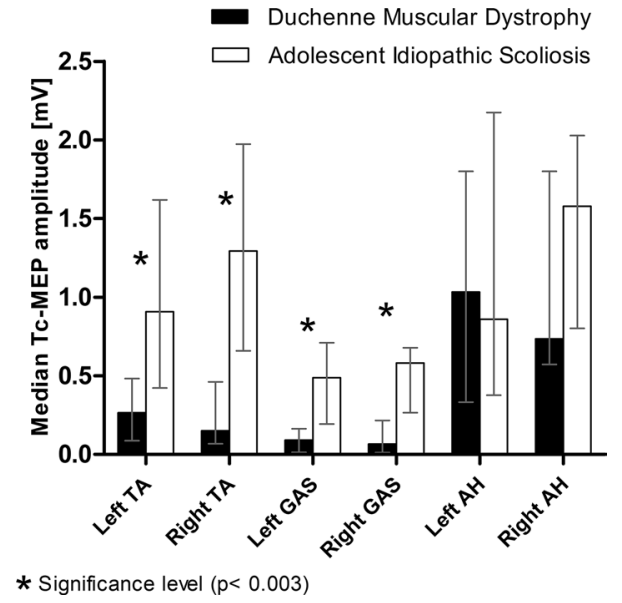

Fig. 1 Median intraoperative Tc-MEP amplitudes. The $\mathrm{x}$-axis denotes the different muscles used for Tc-MEP, and the y-axis denotes the median amplitude in $\mathrm{mV}$. The median intraoperative Tc-MEP amplitudes for patients with DMD (black bars) and AIS (white bars) are shown. Median intraoperative Tc-MEP amplitudes of the TA and GAS muscles were significantly higher in patients with AIS. The abductor hallucis muscles had similar median amplitudes in both patients with DMD and patients with AIS. Tc-MEP Transcranial Electrical Stimulation Motor Evoked Potential; $N$ number; $T A$ Tibialis Anterior muscle; GAS Gastrocnemius muscle; $A H$ Abductor Hallucis muscle

test. The AH left and AH right were not significantly different ( $p=0.567$ and $p=0.325$ respectively).

SSEPs were elicitable from at least one leg or arm in 14 DMD patients (93.3\%). SSEP data were missing in one patient $(6.7 \%)$ due to a technical problem. SSEPs were always elicitable in all patients with AIS.

Non-limb muscles were monitored as well in 11 patients with DMD. Muscles included left and right sphincter muscles and the left and right rectus abdominis muscle. In six patients (54.5\%), Tc-MEP was elicitable in both left and right sphincter muscles and in one patient $(9.1 \%) \mathrm{Tc}-$ MEP was elicitable in the left and right rectus abdominis muscles. In the remaining four patients (36.4\%), Tc-MEP was elicitable in all four muscles.

\section{Preoperative CMAP and TMS}

Preoperative CMAP and TMS measurements were taken to assess if the patients were eligible for monitoring [19]. TMS was elicitable in 14 patients (93.3\%) with DMD. In one patient, the TMS was not elicitable. The CMAP of the tibialis anterior (TA) muscle was elicitable in 12 patients $(80.0 \%)$ with DMD. CMAP data were not available for three patients (20.0\%) with DMD; in two patients, the CMAP was not performed and one patient was too anxious to be measured. In 12 patients (80.0\%) with DMD, both TMS and CMAP were elicitable, and therefore, the TMS/CMAP ratio could be calculated.

The TMS/CMAP ratio could be calculated for all AIS patients $(100.0 \%)$.

The median CMAPs of both the patients with DMD and AIS are shown in Fig. 2A. The highest CMAP of either the left or right TA muscle was used. The median CMAP of the patients with DMD was $1.75 \mathrm{mV}$ (IQR 1.20-3.05), while the median CMAP of the patients with AIS was $6.80 \mathrm{mV}$ (IQR 5.70-8.40). The median CMAP is significantly different $(P<0.001)$ as evidenced by the Mann-Whitney U test.

The TMS/CMAP ratio was obtained using the highest CMAP and TMS of either the left or right TA muscle. A similar median was found $(p=0.126)$ for both patients with DMD (0.67 IQR 0.39-1.00) and AIS (0.47 IQR 0.31-0.69) (Fig. 2B). Figure 2C shows similar median Tc-MEP/CMAP ratios for the left or right TA muscles for both groups. The median ratio in the patients with DMD was 0.22 (IQR 0.06-0.39) and in the patients with AIS was 0.19 (IQR 0.11-0.53). The Tc-MEP/CMAP ratios were not significantly different $(p=0.792)$ as evidenced by the Mann-Whitney U test.
Table 2 Median intraoperative Tc-MEP amplitudes (mV)

\begin{tabular}{llllllll}
\hline & \multicolumn{3}{l}{ Duchenne muscular dystrophy } & & \multicolumn{3}{l}{ Adolescent idiopathic scoliosis } \\
\cline { 2 - 3 } Muscle & $N$ & median $(\mathrm{mV})$ & IQR $(\mathrm{mV})$ & & $N$ & median $(\mathrm{mV})$ & IQR $(\mathrm{mV})$ \\
\hline TA left & 15 & 0.27 & $0.09-0.48$ & & 15 & 0.91 & $0.42-1.62$ \\
TA right & 15 & 0.15 & $0.07-0.46$ & & 15 & 1.29 & $0.66-1.97$ \\
GAS left & 15 & 0.09 & $0.01-0.16$ & & 15 & 0.49 & $0.19-0.71$ \\
GAS right & 15 & 0.07 & $0.01-0.22$ & & 15 & 0.58 & $0.27-0.68$ \\
AH left & 15 & 1.03 & $0.33-1.80$ & & 15 & 0.86 & $0.38-2.17$ \\
AH right & 15 & 0.74 & $0.57-1.80$ & & 15 & 1.58 & $0.80-2.03$ \\
\hline
\end{tabular}

$I Q R$ interquartile range; $T c-M E P$ Transcranial Electrical Stimulation Motor Evoked Potential; $N$ number; $S D$ Standard Deviation; TA Tibialis Anterior muscle; GAS Gastrocnemius muscle; $A H$ Abductor Hallucis muscle 

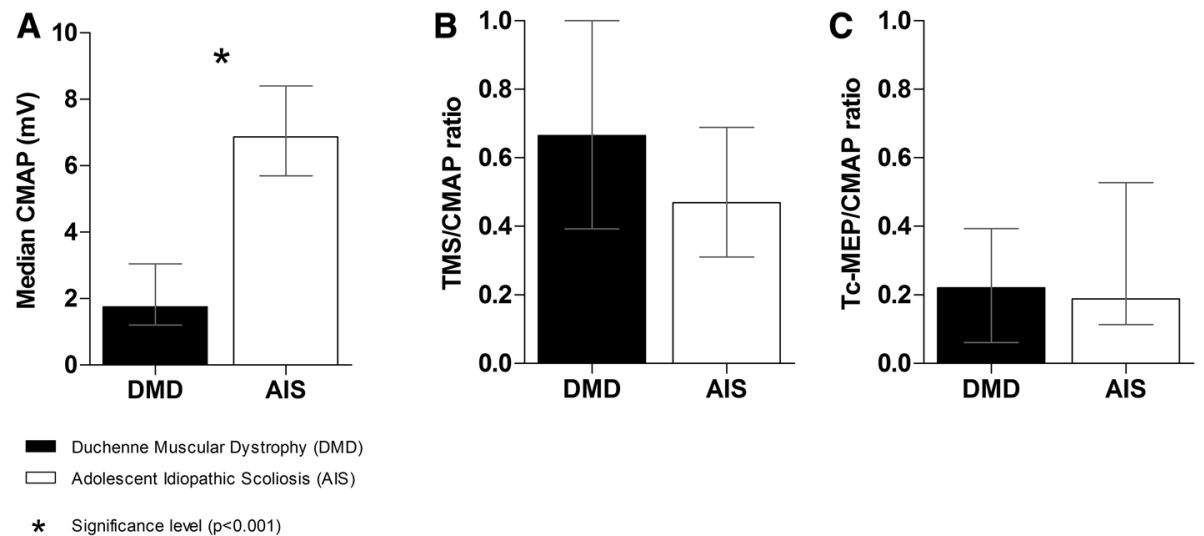

Fig. 2 A. Median Compound Muscle Action Potential; B TMS/ CMAP ratio; C. Tc-MEP/CMAP ratio. Median CMAP, TMS/CMAP ratio and Tc-MEP/CMAP ratios of patients with DMD (black bars) and patients with AIS (white bars). A The median CMAP is significantly higher in patients with AIS than in patients with DMD $(P<0.001)$. B The TMS/CMAP ratio is similar in both patients with

\section{Discussion}

In this study, we investigated the feasibility of IONM and the cortical excitability in patients with DMD and scoliosis and compared the findings with those from patients with AIS. Our study had two main findings, namely that (1) AH muscle amplitudes are similar in patients with DMD and AIS and (2) the cortical excitability seems to be similar for patients with DMD and AIS.

The different degree of fatty infiltration in the different leg muscles in patients with DMD may account for the first main finding that the AH muscles showed similar amplitudes in both groups. Loss of muscle tissue in DMD patients with subsequent fat infiltration results in lower Tc-MEP amplitudes, since there is less muscle to elicit. Wokke et al. showed in an MRI study, in which they investigated the fatty infiltration of the leg muscles in patients with DMD that lower leg muscles have lower fat percentages as compared with the upper leg muscles [20]. Together with another study, they demonstrated high fatty infiltration percentages and scores for the GAS and TA muscles, explaining our lower amplitude findings in the GAS and TA muscles [20, 21]. Unfortunately, in these studies the fat percentages of the AH muscles were not measured. However, our findings are concordant with the fact that distal muscles are involved later than proximal muscles in patients with DMD.

In support of our first main finding, it is important to evaluate how to monitor patients with DMD in the most feasible and reliable way in order to decrease the risk of surgically induced neurological damage. Kim et al. state that the TA muscles are the most reliable muscles to detect neurological damage using Tc-MEPs in patients that underwent spinal surgery [22]. For patients with DMD, this might also be the
DMD and patients with AIS $(p=0.126)$. C The Tc-MEP/CMAP ratio is similar in both patients with DMD and patients with AIS $(p=0.792)$. TMS Transcranial Magnetic Stimulation; CMAP Compound Muscle Action Potential; Tc-MEP Transcranial Electrical Stimulation Motor Evoked Potential; $n$ Number; DMD Duchenne Muscular Dystrophy; AIS Adolescent Idiopathic Scoliosis

case, but the AH muscles could correspondingly contribute to more reliable IONM measurements, especially because of the later development of the fatty infiltration in the $\mathrm{AH}$ muscles in these patients.

SSEPs were elicitable in all patients with DMD for whom data were available (93.3\%), and there were no alerts of significant SSEP amplitude declines. Similarly to our study, Hermanns et al. investigated the reliability of SSEPs in 54 patients with neuromuscular scoliosis. They demonstrated that $88.9 \%$ of the SSEPs were recordable and reproducible leading to their conclusion that SSEPs are as sensitive and specific for patients with neuromuscular scoliosis as for patients with AIS [23].

Monitoring non-limb muscles may be useful as escape muscles when Tc-MEP is not elicitable in few or no leg muscles in patients with DMD. Our results show that nonlimb muscles, consisting of the sphincter muscles and the rectus abdominis muscles, can be used for monitoring. In tethered cord surgery, Tc-MEP of the sphincter muscles has been used frequently and has proven its value [24]. However, in scoliosis correction surgery, its feasibility and reliability have not been investigated properly yet and should therefore be further examined.

Our second main finding is that the cortical excitability was similar for patients with DMD and patients with AIS. It is thought that the presence of dystrophin in the CNS in patients with DMD could have an effect on TMS and Tc-MEP measurements [25]. In our study, preoperative CMAP amplitudes were significantly lower in patients with DMD and this could have been explained by muscle weakness and/or the CNS involvement of dystrophin. However, when TMS/CMAP ratios and Tc-MEP/CMAP ratios were calculated, similar values were found in patients with 
DMD and patients with AIS. This observation suggests that the possible cortical alterations in patients with DMD have no substantial influence on the cortical excitability when using TMS or Tc-MEP. Thus, both when patients are awake (TMS) and under anesthesia (Tc-MEP), the cortical excitability seems not to be different in patients with DMD and patients with AIS.

Our TMS findings are in agreement with the findings of Yayla et al., who stated that TMS excitability in patients with DMD did not reveal abnormalities caused by the disorder, and that TMS and CMAP measurements did not reveal any CNS involvement.

Although this study has limitations, it involves one of the larger cohorts of patients with DMD that underwent scoliosis surgery. However, the sample size is still too small to assess the reliability of IONM in patients with DMD.

In conclusion, routine multimodality IONM of spinal cord sensory and motor function is feasible during surgical correction of scoliosis in patients with DMD, particularly when Tc-MEPs are recorded from the AH muscle. The reliability and feasibility of escape muscles should be further examined. Although CMAP amplitudes were significantly lower in the DMD group, the cortical excitability was similar in patients with DMD and in patients with AIS.

\section{Compliance with ethical standards}

Conflict of interest ARA: The research group/department to which he belongs has received grants and funding from The Medicines Company, Carefusion/Becton Dickinson. He has performed sponsor-initiated phase 1 studies (payment to institution) for The Medicines Company and Rigel; and he has performed paid consultancy work (payment to institution) for Johnson \& Johnson, Carefusion, The Medicines Company, Philips and Ever Pharma. He is an editor of the British Journal of Anaesthesia. All other authors report no conflicts of interest or competing interests in any concept discussed in this article.

Open Access This article is licensed under a Creative Commons Attribution 4.0 International License, which permits use, sharing, adaptation, distribution and reproduction in any medium or format, as long as you give appropriate credit to the original author(s) and the source, provide a link to the Creative Commons licence, and indicate if changes were made. The images or other third party material in this article are included in the article's Creative Commons licence, unless indicated otherwise in a credit line to the material. If material is not included in the article's Creative Commons licence and your intended use is not permitted by statutory regulation or exceeds the permitted use, you will need to obtain permission directly from the copyright holder. To view a copy of this licence, visit http://creativecommons.org/licenses/by/4.0/.

\section{References}

1. Pane M, Mazzone ES, Fanelli L et al (2014) Reliability of the performance of upper limb assessment in Duchenne muscular dystrophy. Neuromuscul Disord 24:201-206. https:// doi.org/10.1016/j.nmd.2013.11.014

2. Lebel DE, Corston JA, McAdam LC et al (2013) Glucocorticoid treatment for the prevention of scoliosis in children with Duchenne muscular dystrophy: long-term follow-up. J Bone Joint Surg Am 95:1057-1061. https://doi.org/10.2106/JBJS.L.01577

3. Birnkrant DJ, Bushby K, Bann CM, et al (2018) Review diagnosis and management of Duchenne muscular dystrophy, part 1: diagnosis, and neuromuscular, rehabilitation, endocrine, and gastrointestinal and nutritional management. 251. https://doi.org/10.1016/ S1474-4422(18)30024-3

4. Suk KS, Lee BH, Lee HM et al (2014) Functional outcomes in Duchenne muscular dystrophy scoliosis: comparison of the differences between surgical and nonsurgical treatment. J Bone Jt Surg Am 96:409-415. https://doi.org/10.2106/JBJS.M.00777

5. Arun R, Srinivas S, Mehdian SMH (2010) Scoliosis in Duchenne's muscular dystrophy: a changing trend in surgical management : a historical surgical outcome study comparing sublaminar, hybrid and pedicle screw instrumentation systems. Eur Spine J 19:376-383. https://doi.org/10.1007/s00586-009-1163-x

6. Berbrayer D (2016) Controversies of scoliosis management in Duchenne muscular dystrophy. PM R 8:S151-S152

7. Cheuk DKL, Wong V, Wraige E et al (2015) Surgery for scoliosis in Duchenne muscular dystrophy. Cochrane Database Syst Rev. https://doi.org/10.1002/14651858.CD005375.pub4

8. Takaso M, Nakazawa T, Imura T et al (2010) Surgical management of severe scoliosis with high risk pulmonary dysfunction in Duchenne muscular dystrophy: patient function, quality of life and satisfaction. Int Orthop 34:695-702. https://doi.org/10.1007/ s00264-010-0957-0

9. Dawson EG, Sherman JE, Kanim LE, Nuwer MR (1991) Spinal cord monitoring. results of the scoliosis research society and the European spinal deformity society survey. Spine (Phila Pa 1976) vol 16, p S361-4

10. Fehlings MG, Brodke DS, Norvell DC, Dettori JR (2010) The evidence for intraoperative neurophysiological monitoring in spine surgery. Spine (Phila Pa 1976). https://doi.org/10.1097/ BRS.0b013e3181d8338e

11. Reames DL, Smith JS, Fu K-MG, et al (2011) complications in the surgical treatment of 19,360 cases of pediatric scoliosis. Spine (Phila Pa 1976) vol 36, p 1484-1491. https://doi.org/10.1097/ BRS.0b013e3181f3a326

12. Subramanyam R, Schaffzin J, Cudilo EM et al (2015) Systematic review of risk factors for surgical site infection in pediatric scoliosis surgery. Spine J 15:1422-1431. https://doi.org/10.1016/j. spinee.2015.03.005

13. Kim T-W, Wu K, Black IB (1995) Deficiency of brain synaptic dystrophin in human duchenne muscular dystrophy. Ann Neurol 38:446-449. https://doi.org/10.1002/ana.410380315

14. DiCindio S, Theroux M, Shah S, et al (2003) Multimodality monitoring of transcranial electric motor and somatosensoryevoked potentials during surgical correction of spinal deformity in patients with cerebral palsy and other neuromuscular disorders. Spine (Phila Pa 1976) vol 28, p 1851-5, discussion p 1855-6. https://doi.org/10.1097/01.BRS.0000083202.62956.A8

15. Langeloo D-D, Journée H-L, de Kleuver M, Grotenhuis JA (2007) Criteria for transcranial electrical motor evoked potential monitoring during spinal deformity surgery. Neurophysiol Clin Neurophysiol 37:431-439. https://doi.org/10.1016/j.neucli.2007.07.007

16. Kamerlink JR, Errico T, Xavier S, et al (2010) Major intraoperative neurologic monitoring deficits in consecutive pediatric and adult spinal deformity patients at one institution. Spine (Phila Pa 1976) vol 35, p 240-5. https://doi.org/10.1097/BRS.0b013e3181 c7c8f6

17. Pastorelli F, Di Silvestre M, Vommaro F et al (2015) Intraoperative monitoring of somatosensory (SSEPs) and transcranial 
electric motor-evoked potentials (tce-MEPs) during surgical correction of neuromuscular scoliosis in patients with central or peripheral nervous system diseases. Eur Spine J 24:931-936. https ://doi.org/10.1007/s00586-015-4282-6

18. Hammett TC, Boreham B, Quraishi NA, Mehdian SMH (2013) Intraoperative spinal cord monitoring during the surgical correction of scoliosis due to cerebral palsy and other neuromuscular disorders. Eur Spine J 22:38-41. https://doi.org/10.1007/s0058 6-012-2652-X

19. Glasby MA, Tsirikos AI, Henderson L et al (2017) Transcranial magnetic stimulation in the semi-quantitative, pre-operative assessment of patients undergoing spinal deformity surgery. Eur Spine J 26:2103-2111. https://doi.org/10.1007/s0058 6-016-4737-4

20. Wokke BH, van den Bergen JC, Versluis MJ et al (2014) Quantitative MRI and strength measurements in the assessment of muscle quality in Duchenne muscular dystrophy. Neuromuscul Disord 24:409-416. https://doi.org/10.1016/j.nmd.2014.01.015

21. Torriani M, Townsend E, Thomas BJ, et al Lower leg muscle involvement in Duchenne muscular dystrophy: an MR imaging and spectroscopy study. https://doi.org/10.1007/s0025 6-011-1240-1
22. Kim S-M, Yang H, Park S-B et al (2012) Pattern-specific changes and discordant prognostic values of individual leg-muscle motor evoked potentials during spinal surgery. Clin Neurophysiol 123:1465-1470. https://doi.org/10.1016/j.clinph.2011.11.035

23. Hermanss H, Lipfert P, Meier S, Jetzek-Zader M, Krauspe R, Stevens M (2007) Cortical somatosensory-evoked potentials during spine surgery in patients with neuromuscular and idiopathic scoliosis under propofol-remifentanil anaesthesia. Br J Anaesth 98:326-335

24. Sala F, Squintani G, Tramontano V et al (2013) Intraoperative neurophysiology in tethered cord surgery: techniques and results. https://doi.org/10.1007/s00381-013-2188-3

25. Di LV, Restuccia D, Servidei S et al (1998) Functional involvement of cerebral cortex in Duchenne muscular dystrophy. Muscle Nerve 21:662-664

Publisher's Note Springer Nature remains neutral with regard to jurisdictional claims in published maps and institutional affiliations. 